\title{
Fiber-based polarization-sensitive Mueller-matrix optical coherence tomography with continuous source polarization modulation
}

Shuliang Jiao, Tseng-Ming Hsieh, Jun Ai, Milos Todorovic, George Stoica, et al.

Shuliang Jiao, Tseng-Ming Hsieh, Jun Ai, Milos Todorovic, George Stoica, Lihong V. Wang, "Fiber-based polarization-sensitive Mueller-matrix optical coherence tomography with continuous source polarization modulation," Proc. SPIE 5316, Coherence Domain Optical Methods and Optical Coherence Tomography in Biomedicine VIII, (1 July 2004); doi: 10.1117/12.531022 


\title{
Fiber-based polarization-sensitive Mueller-matrix optical coherence tomography with continuous source polarization modulation
}

\author{
Shuliang Jiao ${ }^{\mathrm{a}}$, Tseng-Ming Hsieh ${ }^{\mathrm{a}}$, Jun $\mathrm{Ai}^{\mathrm{a}}$, Milos Todorovic ${ }^{\mathrm{a}}$, George Stoica ${ }^{\mathrm{b}}$, Lihong V. Wang ${ }^{\mathrm{a}}$ \\ ${ }^{a}$ Optical Imaging Laboratory, Department of Biomedical Engineering, Texas A\&M University, \\ 3120 TAMU, College Station, TX 77843-3120 \\ ${ }^{\mathrm{b}}$ Department of Pathobiology, Texas A\&M University, College Station, TX 77843-5547
}

\begin{abstract}
A novel fiber-based Mueller-matrix optical coherence tomography system is demonstrated for acquiring polarization images of biological tissues in vivo. The system features a single broadband source, a rapid scanning optical delay line, and an electro-optical polarization modulator that modulates the polarization states of the source light continuously. A frame of a $200 \times 1515$-pixel 2D image can be acquired in half a second. The Jones matrix of a sample is calculated from two frequency components--the A-scan carrier frequency component and the beat frequency component between the modulation frequency and the carrier frequency. For samples having negligible diattenuation, the Jones matrix can be calculated from a single measurement of either the horizontal or the vertical interference signal. The system was first validated by imaging standard polarization elements and then applied to imaging biological samples.
\end{abstract}

Keywords: optical coherence tomography, Mueller-matrix, Jones Matrix polarization

\section{INTRODUCTION}

As a branch of polarization-sensitive optical coherence tomography (PS-OCT), ${ }^{1}$ Mueller-matrix OCT has been developed to fully characterize the polarization properties of biological tissues. ${ }^{2-6}$ Because of the interference-based heterodyne detection scheme in OCT, a scattering sample acts as a nondepolarizing medium. In a nondepolarizing medium, the Mueller matrix and the Jones matrix are equivalent and can be transformed from each other. The polarization properties, such as retardance, orientation, ellipticity, diattenuation, etc., of biological tissues can be computed from either the Jones matrix or the Mueller matrix.

To measure a Jones Matrix, at least two independent incident polarizations states are needed. In our previous versions of Mueller-matrix OCT, two independent sources with orthogonal polarization states were used to calculate the Jones Matrix of a sample with a single measurement. In this paper, we demonstrate a novel Mueller-matrix OCT system that employs a single super-luminescent diode followed by a polarization modulator to continuously modulate the incident polarization states of the interferometer.

\section{EXPERIMENTAL SET-UP}

A schematic of the experimental system is shown in figure 1. The SLD light source has a center wavelength of 1.3 um, a $3 \mathrm{~dB}$ bandwidth of $70 \mathrm{~nm}$ and output power of $10 \mathrm{~mW}$. A linear polarizer sets the polarization state of the SLD source to vertical before it hits the polarization modulator (Conoptics), which continuously modulates the incident polarization state at $>100 \mathrm{kHz}$ to ensure that multiple cycles of polarization states are applied to each layer (corresponding to the depth resolution) of the depth scan. The fast axis of the polarization modulator is oriented at $45^{\circ}$. A linear polarizer (LP) oriented at $-45^{\circ}$ is placed in the reference arm to control the reference polarization state. The reference arm and the sample arm are composed of single mode optical fibers. A grating-based rapid scanning optical delay line (RSOD) is used for the depth scan. The combined sample and reference light is split into horizontal and vertical polarization components by a polarizing beam splitter (PBS). The two orthogonal polarization components of the interference signal are detected by two photodiodes - PDH and PDV- respectively. A 12-bit DAQ card is used to digitize the detected analog signal for computer processing. A frame of a $200 \times 1515$-pixel image can be acquired in half a second. 


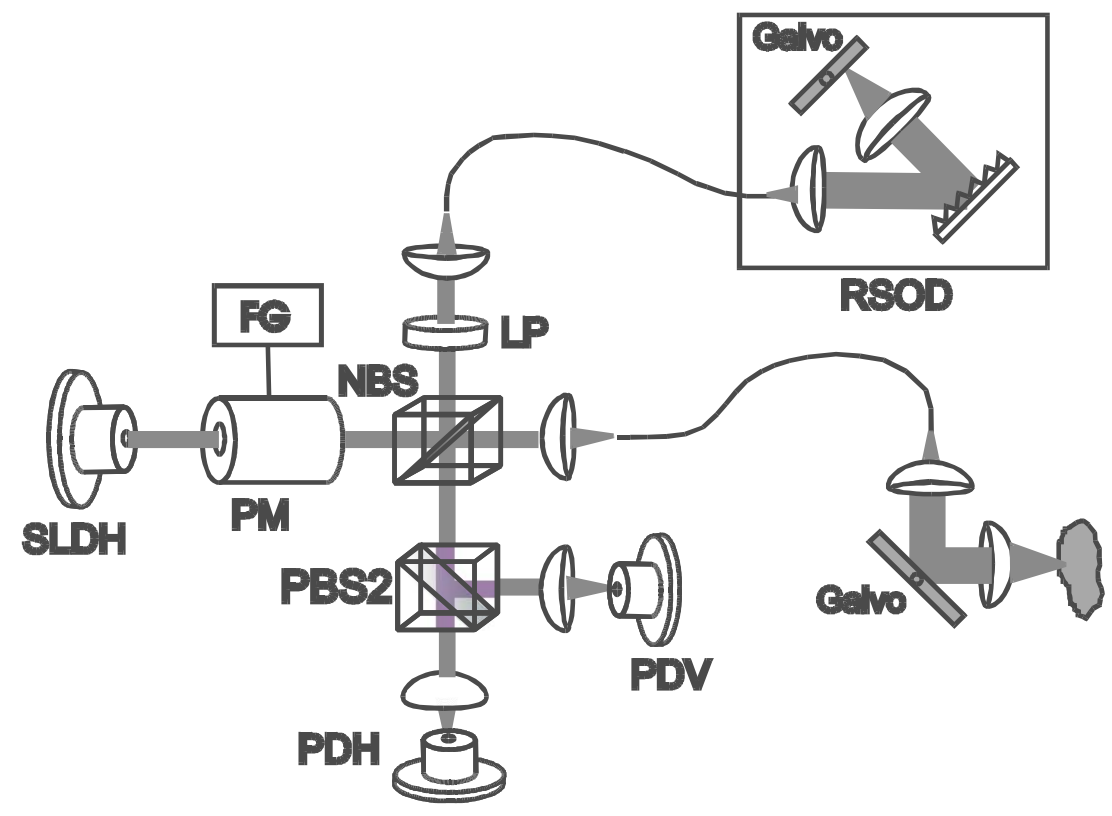

Figure 1. Schematic of the experimental system. SLDH: SLD, horizontally polarized; PM: polarization modulator; FG: function generator; NBS: non-polarizing beam splitter; PBS2: polarizing beam splitter; SMF: single-mode optical fiber; PDH and PDV: photodiodes $\mathrm{H}$ and $\mathrm{V}$ polarization. RSOD: rapid scanning optical delay line; LP: $45^{\circ}$ linear polarizer.

\section{JONES MATRIX CALCULATION}

The Jones matrix of the polarization modulator $\mathrm{J}_{\mathrm{m}}$ can be expressed as

$$
\mathbf{J}_{m}(\varphi, \pi / 4)=\left[\begin{array}{cc}
\cos \varphi / 2 & i \sin \varphi / 2 \\
i \sin \varphi / 2 & \cos \varphi / 2
\end{array}\right]
$$

where $\varphi$ is the phase retardation of the polarization modulator. The incident Jones vector $E_{i}$ for both the sample and the reference arms can be expressed as

$$
\mathbf{E}_{i}=\left[\begin{array}{c}
i \sin (\varphi / 2) \\
\cos (\varphi / 2)
\end{array}\right]
$$

The output Jones vector $E_{o}$ of the sample arm can be obtained from $\mathbf{E}_{o}=\mathbf{J}_{T} \mathbf{E}_{i}$ as

$$
\mathbf{E}_{o}=\left[\begin{array}{c}
E_{o x} \\
E_{o y}
\end{array}\right]=\left[\begin{array}{c}
J(1,2) \cos (\varphi / 2)+i J(1,1) \sin (\varphi / 2) \\
J(2,2) \cos (\varphi / 2)+i J(2,1) \sin (\varphi / 2)
\end{array}\right],
$$

where $\mathbf{J}_{T}=\left[\begin{array}{ll}J(1,1) & J(1,2) \\ J(1,2) & J(2,2)\end{array}\right]$ is the round-trip Jones matrix of the sample arm. The Jones matrix of the reference arm $\mathbf{J}_{\text {ref }}$ can be expressed as

$$
\mathbf{J}_{\text {ref }}=1 / 4\left[\begin{array}{cc}
1 & -1 \\
-1 & 1
\end{array}\right] \mathbf{J}_{r f 2}\left[\begin{array}{cc}
1 & -1 \\
-1 & 1
\end{array}\right],
$$

where $\mathbf{J}_{r f 2}$ is the round-trip Jones matrix of the single-mode optical fiber in the reference arm. The output Jones vector of the reference arm can be obtained from $\mathbf{E}_{r e f}=\mathbf{J}_{r e f} \mathbf{E}_{i}$. 


$$
\mathbf{E}_{r e f}=\left[\begin{array}{c}
E_{r e f x} \\
E_{r e f y}
\end{array}\right]=\left[\begin{array}{c}
E_{r h} \\
-E_{r v}
\end{array}\right] \exp (-i \varphi / 2),
$$

where $E_{r h}$ and $E_{r v}$ have the same phase and are functions of $\mathbf{J}_{r f 2}$. We then have the detected intensities in the horizontal and vertical channels:

$$
\begin{aligned}
& I_{x}=\left|E_{\text {refx } x} \exp \left(i \bar{k} z_{r}\right)+E_{o x} \exp \left(i \bar{k} z_{s}\right)\right|^{2}=I_{x 0}+\tilde{I}_{x} \\
& I_{y}=\left|E_{\text {refy }} \exp \left(i \bar{k} z_{r}\right)+E_{\text {oy }} \exp \left(i \bar{k} z_{s}\right)\right|^{2}=I_{y 0}+\tilde{I}_{y}
\end{aligned}
$$

where $\bar{k}$ is the center wave number of the light source in the vacuum; $z_{s}$ and $z_{r}$ are the optical path lengths of the sample and reference arms, respectively; $I_{x o}$ and $\tilde{I}_{x}\left(I_{y o}\right.$ and $\left.\tilde{I}_{y}\right)$ are the non-interference and interference parts of the signal in the horizontal channel (vertical channel), respectively. The interference parts can be further processed as

$$
\begin{aligned}
& \tilde{I}_{x}=\left|E_{r h}\right| \operatorname{Re}\left\{[J(1,1)+J(1,2)] \exp \left[-i\left(\bar{k} z+\varphi_{r}-\varphi\right)\right]+[J(1,2)-J(1,1)] \exp \left[-i\left(\bar{k} z+\varphi_{r}\right)\right]\right\} \\
& \tilde{I}_{y}=\left|E_{r v}\right| \operatorname{Re}\left\{[J(1,2)+J(2,2)] \exp \left[-i\left(\bar{k} z+\varphi_{r}+\pi-\varphi\right)\right]\right\}+ \\
& \\
& \quad\left|E_{r v}\right| \operatorname{Re}\left\{[J(2,2)-J(1,2)] \exp \left[-i\left(\bar{k} z+\varphi_{r}+\pi\right)\right]\right\}
\end{aligned}
$$

where $\varphi_{r}$ is the phase of $E_{r h}$ and $E_{r v} ; \mathrm{z}$ is the path length difference between the reference and sample arms. $\tilde{I}_{x}$ and $\tilde{I}_{y}$ can be further expanded as

$$
\begin{aligned}
\tilde{I}_{x}= & \left|E_{r h}\right| \cdot|J(1,2)-J(1,1)| \cos \left(\bar{k} z+\varphi_{r}-\varphi_{x 1}\right) \\
& +\left|E_{r h}\right| \cdot|J(1,1)+J(1,2)| \cdot\left[\cos \left(\bar{k} z+\varphi_{r}-\varphi_{x 2}\right) \cos \varphi+\sin \left(\bar{k} z+\varphi_{r}-\varphi_{x 2}\right) \sin \varphi\right], \\
\tilde{I}_{y} & =\left|E_{r v}\right| \cdot|J(2,2)-J(1,2)| \cos \left(\bar{k} z+\varphi_{r}+\pi-\varphi_{y 1}\right) \\
& +\left|E_{r v}\right| \cdot|J(1,2)+J(2,2)| \cdot\left[\cos \left(\bar{k} z+\varphi_{r}+\pi-\varphi_{y 2}\right) \cos \varphi+\sin \left(\bar{k} z+\varphi_{r}+\pi-\varphi_{y 2}\right) \sin \varphi\right]
\end{aligned}
$$

where $\varphi_{x 1}, \varphi_{x 2}, \varphi_{y 1}$, and $\varphi_{y 2}$ are the phases of $J(1,2)-J(1,1), J(1,1)+J(1,2), J(2,2)-J(1,2)$, and $J(1,2)+J(2,2)$, respectively. When the polarization modulator is driven by a sinusoidal wave, i.e., $\varphi=A_{0} \sin \omega_{m} t$, where $\omega_{m}$ is the frequency of the driving wave, we have

$$
\begin{aligned}
& \sin \varphi=\sum_{l=0}^{\infty} 2 J_{2 l+1}\left(A_{0}\right) \sin \left[(2 l+1) \omega_{m} t\right] \\
& \cos \varphi=J_{0}\left(A_{0}\right)+\sum_{l=1}^{\infty} 2 J_{2 l}\left(A_{0}\right) \cos \left[(2 l) \omega_{m} t\right]
\end{aligned}
$$

where $J_{0}, J_{2 l+1}$, and $J_{2 l}$ are the Bessel functions of the first kind of the order of $0,21+1$, and 21. When $\mathrm{A}_{0}=2.405$, $J_{0}\left(A_{0}\right)=0$. We then have 


$$
\begin{aligned}
\tilde{I}_{x}= & \left|E_{r h}\right| \cdot|J(1,2)-J(1,1)| \cos \left(\bar{k} z+\varphi_{r}-\varphi_{x 1}\right) \\
& +\left|E_{r h}\right| \cdot|J(1,1)+J(1,2)| \cos \left(\bar{k} z+\varphi_{r}-\varphi_{x 2}\right) \sum 2 J_{2 l}(2.405) \cos \left[(2 l) \omega_{m} t\right] \\
& +\left|E_{r h}\right| \cdot|J(1,1)+J(1,2)| \sin \left(\bar{k} z+\varphi_{r}-\varphi_{x 2}\right) \sum 2 J_{2 l+1}(2.405) \sin \left[(2 l+1) \omega_{m} t\right], \\
\tilde{I}_{y} & =\left|E_{r v}\right| \cdot|J(2,2)-J(1,2)| \cos \left(\bar{k} z+\varphi_{r}+\pi-\varphi_{y 1}\right) \\
& +\left|E_{r v}\right| \cdot|J(1,2)+J(2,2)| \cos \left(\bar{k} z+\varphi_{r}+\pi-\varphi_{y 2}\right) \sum 2 J_{2 l}(2.405) \cos \left[(2 l) \omega_{m} t\right] \\
& +\left|E_{r v}\right| \cdot|J(1,2)+J(2,2)| \sin \left(\bar{k} z+\varphi_{r}+\pi-\varphi_{y 2}\right) \sum 2 J_{2 l+1}(2.405) \sin \left[(2 l+1) \omega_{m} t\right] .
\end{aligned}
$$

The elements of $\mathbf{J}_{T}$ can be calculated from two frequency components in eq (10), e.g.

$$
\begin{aligned}
J(1,1) & =\left[-\tilde{I}_{x}^{*}(\bar{k} z)+\tilde{I}_{x}\left(\omega_{m} t-\bar{k} z\right) / J_{1}(2.405)\right] /\left|E_{r h}\right|, \\
J(2,2) & =\left[-\tilde{I}_{y}^{*}(\bar{k} z)-\tilde{I}_{y}\left(\omega_{m} t-\bar{k} z\right) / J_{1}(2.405)\right] /\left|E_{r v}\right|, \\
J(1,2) & =\left[\tilde{I}_{x}^{*}(\bar{k} z)+\tilde{I}_{x}\left(\omega_{m} t-\bar{k} z\right) / J_{1}(2.405)\right] /\left|E_{r h}\right|, \text { or } \\
J(1,2) & =\left[\tilde{I}_{y}^{*}(\bar{k} z)-\tilde{I}_{y}\left(\omega_{m} t-\bar{k} z\right) / J_{1}(2.405)\right] /\left|E_{r v}\right| .
\end{aligned}
$$

As shown above, the Jones matrix of the sample arm can be calculated with two frequency terms in the horizontal and vertical detection channels. One frequency is determined by the depth scan (the carrier frequency); the other one is the beat frequency between the modulation and the carrier. Other frequency terms can also be used in the calculation. Please note, $\mathrm{J}(1,2)$ can be calculated from either detection channel. This relationship can be used to balance the two detection channels when the two detectors are not identical.

If the diattenuation of a sample is negligible, then $J(2,2)=J(1,1)^{*}$. In this case, we see from Eq. (11) that $\mathbf{J}_{T}$ can be completely calculated from either $\tilde{I}_{x}$ or $\tilde{I}_{y}$. As a result, only one detection channel is needed to measure the roundtrip Jones matrix.

\section{SYTEM CALIBRATION AND EXPERIMENTAL RESULTS}

The system was first tested with a standard polarization element - a quarter-wave ( $\lambda / 4)$ plate - cascaded by a mirror as the sample. The fast axis of the wave plate was set at various orientations. The polarization modulator was driven by a $140 \mathrm{kHz}$ sinusoidal wave. To verify the amplitude of the driving wave, we placed a mirror between the non-polarizing beam splitter and the entrance of the sampling fiber and measured the ratio of the intensities of the second and the fourth harmonics of the modulation frequency of the modulator in both detection channels to ensure that:

$$
\left|I_{x}\left(2 \omega_{m}\right)\right| /\left|I_{x}\left(4 \omega_{m}\right)\right|=\left|I_{y}\left(2 \omega_{m}\right)\right| /\left|I_{y}\left(4 \omega_{m}\right)\right|=J_{2}(2.405) / J_{4}(2.405)=6.667 \text {. }
$$

The measured Jones matrix of the sampling fiber was used to eliminate the polarization effect of the sampling fiber. The round-trip retardation and the orientation of the fast axis of the wave plate were calculated. The results match the expected values satisfactorily, which validates the experimental system.

The system was then used to image biological samples of porcine tendon, human finger tip and human thin skin in vivo. The polarization images were extracted from the measured Jones matrices. The intensity images and the retardation images are shown in Fig. 2. 
[a]

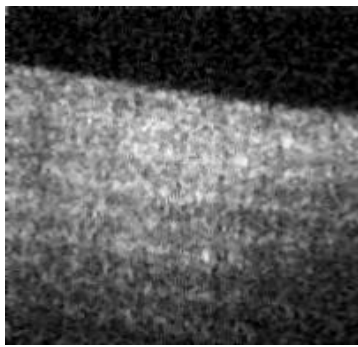

[b]

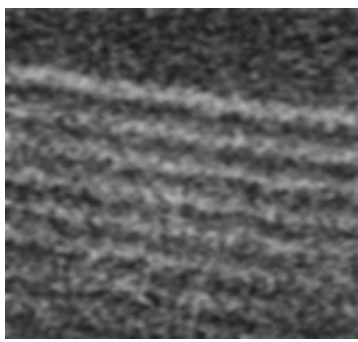

[c]

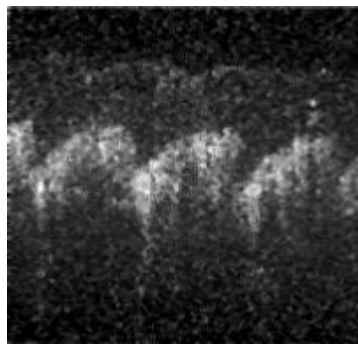

[d]

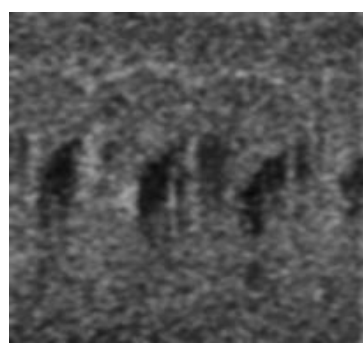

[e]

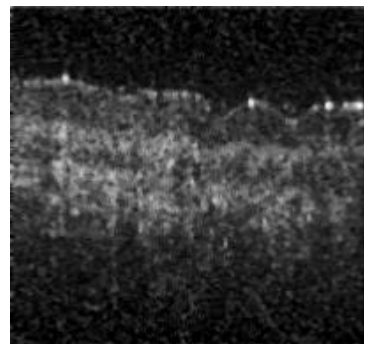

[f]

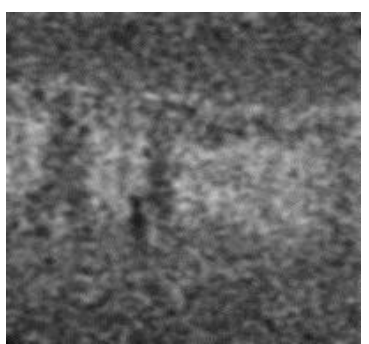

Fig. 2 [a] and [b]: intensity and retardation images of porcine tendon, [c] and [d]: intensity and retardation images of human finger, [e] and [f]: intensity and retardation images of human thin skin.

\section{CONCLUSION}

In conclusion, we have developed a novel fiber-based polarization-sensitive Mueller-matrix optical coherence tomography that enables the acquisition of a round-trip Jones matrix of biological samples using only one light source and a single depth scan. A polarization modulator is used in the source arm to continuously modulate the incident polarization states. The Jones matrix of the sample can be calculated from two frequency terms in two detection channels. When diattenuation is negligible, only a single detection channel and a single measurement are needed to measure the Jones matrix. The system was validated by standard optical elements and applied to imaging biological samples.

\section{ACKNOWLEDGEMENT}

This project was sponsored in part by National Institutes of Health grants R21 EB00319-02 and R01 EB000712, by National Science Foundation grant BES-9734491, and by Texas Higher Education Coordinating Board grant 0005120063-2001. L. V. Wang's email address is LWang@tamu.edu.

\section{REFERENCES}

1. J. F. de Boer, T. E. Milner, M. J. C. van Gemert and J. S. Nelson, "Two dimensional birefringence imaging in biological tissue by polarization-sensitive optical coherence tomography," Optics Letters, 22, 934-936 (1997).

2. S. Jiao, W. Yu, G. Stoica, and L. V. Wang, " Fiber-based Mueller optical coherence tomography", Optics Letters, 28(14), 1206-1208 (2003).

3. S. Jiao, W. Yu, G. Stoica, and L. V. Wang, " Phase-based polarization contrast in Polarization-sensitive Muellermatrix optical coherence tomography and application in burn imaging", Applied Optics, 42(25), September, (2003).

4. S. Jiao and L. V. Wang, "Jones-matrix Imaging of biological tissues with quadraple-channel optical coherence tomography", J. of Biomedical Optics 7(3), 350-358 (2002)

5. S. Jiao and L. V. Wang, "Two-dimensional depth-resolved Mueller matrix of biological tissue measured with double-beam polarization-sensitive optical coherence tomography", Optics Letters 27(2), 101-103(2002).

6. S. Jiao, G. Yao, and L. V. Wang, "Depth-resolved two-dimensional Stokes vectors of backscattered light and Mueller matrices of biological tissue measured with optical coherence tomography", Applied Optics 39(34), 63186324(2000). 